\section{RENAL RECOVERY FOLLOWING USE OF LEFT VENTRICULAR ASSIST DEVICE IN ADVANCED HEART FAILURE PATIENTS}

Tarique Musa*, Colin Chue, Hoong Sern Lim. 'Queen Elizabeth Hospital Birmingham, University Hospital Birmingham NHS Foundation Trust

\subsection{6/heartjnl-2017-311726.20}

Background The National Institute of Health and Care Excellence advocates the use of a Left Ventricular Assist Device (LVAD) as a bridge to transplant, and furthermore as destination therapy in patients who are otherwise ineligible to receive transplantation. Accordingly, the UK has witnessed a sharp increase in the use of LVAD implantation as mechanical circulatory support in patients with advanced heart failure. Heart Failure and kidney disease are significant public health concerns, and given patients with renal dysfunction have largely been excluded from heart failure trials; their optimal management remains unclear.

Aim To assess renal outcomes in patients with advanced heart failure, at both early and mid-term follow-up, following LVAD implantation.

Methods A retrospective single-centre analysis was conducted in 64 consecutive patients implanted with either HeartMate II or HeartMate III continuous-flow LVADs (Thoratec Corp., Pleasanton, California). Renal biochemical profile and estimated glomerular filtration rate (eGFR) was assessed immediately prior to, 30 days and 6 months following LVAD implantation. For the purposes of statistical analysis, stages of chronic kidney disease were defined to reflect the Kidney Disease Outcomes Quality Initiative (Stage 1; GFR 90+, Stage 2; GFR 60-89, Stage 3; GFR 45-59 (KDOQI Stage 3A), Stage 4; 30-44 (KDOQI Stage 3B), Stage 5; GFR 15-29). Patients on dialysis were excluded.

Results 64 patients (age 52.5 \pm 11.5 years, $88 \%$ male, 14\% diabetic, median INTERMACS Profile 3 (IQR 3.00), cardiac output $3.51 \pm 0.99 \mathrm{~L} / \mathrm{min}$ ) were studied immediately prior to LVAD implantation, and subsequently at 30 days $(n=60)$ and 6 months $(n=42)$. The results are summarised in Table 1 .

\begin{tabular}{|c|c|c|c|c|}
\hline & Urea & Creatinine & eGFR & CKD stage \\
\hline Baseline $(n=64)$ & $8.5 \pm 4.65$ & $120.4 \pm 49.54$ & $59.8 \pm 21.13$ & 3.0 (IQR 1.75) \\
\hline 30 day $(n=60)$ & $6.6 \pm 5.53$ & $85.6 \pm 30.02$ & $75.6 \pm 17.31$ & 2.0 (IQR 1.0) \\
\hline$p$ Value & 0.029 & $<0.001$ & $<0.001$ & $<0.001$ \\
\hline 6 month $(n=42)$ & $6.9 \pm 2.93$ & $96.6 \pm 27.88$ & $72.4 \pm 17.38$ & 2.0 (IQR 0.25 ) \\
\hline p Value (vs baseline) & 0.196 & 0.017 & 0.004 & 0.062 \\
\hline
\end{tabular}

Significant statistical improvements were seen in urea, creatinine and CKD stage at 30 days; with the improvement in eGFR from baseline persisting at 6 months. In sub-group analysis, excluding patients with normal baseline renal function (defined as CKD Stage 1, $\mathrm{n}=10$ ), a significant improvement in CKD stage was seen both early (3.0 (IQR 2.0) vs 2.0 (IQR $1.0), \mathrm{p}<0.001$ ) and at mid-term (3.0 (IQR 2.0) vs. 2.0 (IQR 1.0) $\mathrm{p}=0.004)$.

Conclusions Renal impairment in patients with end-stage heart failure prior to LVAD placement is common and reversible. An early and marked improvement in renal biochemistry from baseline, persisting at 6 months, is seen following LVAD implantation, suggesting poor renal perfusion as a primary aetiology. Given the improvement in CKD stage by an average of 1 grade; further work is required to determine whether LVAD is the treatment of choice in those with significant baseline renal dysfunction.

\section{Interventional Cardiology}

\section{A COMPARISON OF CORONARY HAEMODYNAMICS IN 4OCC VERSUS 5OCC INTRA-AORTIC BALLOON PUMPS}

${ }^{1}$ Natalia Briceno*, ${ }^{2}$ Kalpa De Silva, ${ }^{3}$ Matthew Lumley, ${ }^{4}$ Balrik Kailey, ${ }^{5}$ Howard Ellis, ${ }^{1}$ Simon Redwood, 'Divaka Perera. 'King's College London, Guy's and St Thomas' Hospital; ${ }^{2}$ King's College Hospital; ${ }^{3}$ King's College London; ${ }^{4}$ King's College Hospital Foundation Trust; ${ }^{5}$ Guy's and St Thomas' Hospital

\subsection{6/heartjnl-2017-311726.21}

Introduction Randomised trials have questioned the benefit of intra-aortic balloon pump (IABP) counterpulsation in high-risk PCI and shock. A larger capacity balloon has been introduced into routine clinical practice that has been shown to provide greater systolic unloading and diastolic augmentation compared with the standard balloon. Our aim was to investigate whether larger capacity balloons provide a greater augmentation in coronary flow compared with standard capacity balloons.

Methods Seven patients with severe ischaemic cardiomyopathy were studied using a two-treatment, single sequence crossover protocol at the time of elective percutaneous coronary intervention (PCI). Simultaneous coronary pressure and Doppler measurements were undertaken in the target vessel after PCI using a Volcano Combowire, during unassisted and assisted IABP conditions, first with a $40 \mathrm{cc}$ IABP and then, five minutes later, with a 50cc IABP. Measurements were taken with intact autoregulation and with autoregulation temporarily disabled by administration of intracoronary adenosine. Coronary wave intensity analysis was performed to characterise the wave energies associated with balloon counterpulsation. Data are presented as mean $\pm \mathrm{SD}$.

Results Patients were $65( \pm 12)$ years old $(75 \%$ male). Left ventricular ejection fraction was $29 \%( \pm 11 \%)$ with a coronary jeopardy score of $11( \pm 2)$ (maximum possible score $=12$ ). There was no difference between the $40 \mathrm{cc}$ and $50 \mathrm{cc}$ IABP balloons in average peak velocity $\left(50.3 \pm 33.6\right.$ vs. $\left.49.7 \pm 24.2 \mathrm{~cm} \mathrm{~s}^{-1} \mathrm{p}=0.916\right)$, mean distal coronary pressure $(83.4 \pm 16$ vs. $85.2 \pm 20 \mathrm{mmHg}, \mathrm{p}=0.549)$ (see Figure 1), or microvascular resistance $(181.6 \pm 52.9$ vs. 207.5 $\left.\pm 83.4 \mathrm{mmHgcm}^{-1} \mathrm{~s}, \mathrm{p}=0.218\right)$ when autoregulation was disabled. Results were similar during basal assisted conditions. On wave intensity analysis, a late diastolic forward compression wave was identified during IABP support that was not seen during unassisted conditions. The magnitude of the IABP-forward compression wave was numerically greater with the $50 \mathrm{cc}$ balloon during basal conditions, which did not reach statistical significance (1.7 \pm 1.7 vs. $2.8 \pm 3.1, \mathrm{~W} \mathrm{~m}^{-2} \mathrm{~s}^{-2} \times 10^{5}, \mathrm{p}=0.155$ ) (see Figure 2).

Conclusions The larger capacity balloon does not provide greater augmentation in coronary flow or reduction in microvascular resistance compared with the standard balloon during basal or hyperaemic conditions. We did not measure left ventricular afterload or myocardial oxygen demand in this study and cannot exclude a differential effect of the $50 \mathrm{cc}$ balloon on myocardial oxygen supply and demand. 